\title{
Myo-inositol and prostaglandins reverse the glucose inhibition of neural tube fusion in cultured mouse embryos
}

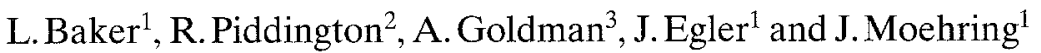 \\ ${ }^{1}$ Department of Pediatrics, School of Medicine and \\ 2 Department of Histology and Embryology, School of Dental Medicine, University of Pennsylvania, Philadelphia, Pennsylvania, \\ ${ }^{3}$ Department of Pediatrics, College of Medicine, University of Illinois, Chicago, Illinois, USA
}

\begin{abstract}
Summary. Neural tube defects in infants of diabetic mothers constitute an important and frequent cause of neonatal mortality/morbidity and long-term chronic handicaps. The mechanism by which normal neural tube fusion occurs is not known. The failure of rostral neural tube fusion seen in mouse embryos incubated in the presence of excess-D-glucose can be significantly prevented by the supplementation of myo-inositol to the culture medium. This protective effect of myo-inositol is reversed by indomethacin, an inhibitor of arachidonic acid metabolism leading to prostaglandin syn-
\end{abstract}

thesis. Prostaglandin $\mathrm{E}_{2}$ added to the culture medium completely protects against the glucose-induced neural tube defect. These data suggest that the failure of neural tube fusion seen in diabetic embryopathy is mediated through a mechanism involving abnormalities in both the myo-inositol and arachidonic acid pathways, resulting in a functional deficiency of prostaglandins at a critical time of neural tube fusion.

Key words: Diabetic embryopathy, myo-inositol, prostaglandins, neural tube, mouse embryos.
Diabetic embryopathy, malformations in infants of diabetic women, represents a continued important complication of diabetes [1]. Serious congenital malformations, primarily involving the central nervous system and the heart, constitute the largest cause of death in the baby of the diabetic mother $[2,3]$.

While several factors have been proposed as important in the mechanism producing diabetic embryopathy [4-10], these hypotheses concerning diabetic embryopathy have, with some exceptions $[11,12]$, tended to remain separate from those mechanisms involving abnormalities in intracellular myo-inositol (MI) and phosphatidylinositol metabolism which have been proposed for diabetic abnormalities in nerve, retina and kidney [1316].

We have previously shown [17] that arachidonic acid supplementation significantly protects against glucose-induced failure of neural tube fusion in cultured mouse embryos. These observations suggested that the arachidonic acid cascade leading to prostaglandin synthesis may be critically involved in the process of normal neural tube fusion.

In this paper, we test whether MI and prostaglandins can reverse the glucose-induced inhibition of neural tube fusion, thus implying that abnormalities in both MI and prostaglandin pathways may play important roles in the mechanism of diabetic embryopathy.

\section{Materials and methods}

Mice of the highly inbred B10.A strain (B10.A/SgSnJ H-2 ${ }^{\mathrm{a}} \mathrm{T1} \mathrm{a}^{\mathrm{a}}$, The Jackson Laboratory Bar Harbor, ME, USA) were used for the MI and indomethacin studies. However, the serious fire at the Jackson Laboratory precluded continued use of this strain. Mice of the random bred CD-1 strain (Crl:CD-1(ICR) BR, Swiss; Charles River Breeding Laboratories Wilmington, MA, USA) were used for the prostaglandin experiments. Both strains show similar control rates of rostral neural tube fusion in the presence of L-glucose and identical inhibitory responses to D-glucose.

For these studies, $\mathrm{D}$ - and $\mathrm{L}$-glucose were used in a concentration of $44 \mathrm{mmol} / 1$. This concentration of D-glucose was chosen not because of any relevance to the glucose levels seen in human diabetic pregnancies, but because it causes rostral neural tube fusion to occur only one-third of the time and thus provides a clear background against which one can judge the protective effects of any intervention. L-glucose at $44 \mathrm{mmol} / \mathrm{l}$ permits neural tube fusion in approximately three-fourths of the cultured embryos. Despite this significant difference in neural tube fusion with D- and L-glucose, growth of embryos, as measured by crown-rump length, is the same with either treatment. The increased number of neural tube malformations in medium containing D-glucose does not appear to be the result of growth delay. 
Table 1. Effects of myo-inositol (MI) and MI plus indomethacin on neural tube fusion in glucose treated mouse embryos in culture

\begin{tabular}{|c|c|c|c|}
\hline & \multirow{2}{*}{$\begin{array}{l}\text { Embryos, total } \\
(n)\end{array}$} & \multicolumn{2}{|c|}{ Fused neural tubes } \\
\hline & & $\overline{(n)}$ & $\%$ \\
\hline $\begin{array}{l}\text { Control (L-glucose, } \\
44 \mathrm{mmol} / \mathrm{l})\end{array}$ & 97 & 75 & 77 \\
\hline D-glucose $(44 \mathrm{mmol} / \mathrm{l})$ & 92 & $32^{\mathrm{a}}$ & 35 \\
\hline $\begin{array}{l}\text { D-glucose }(44 \mathrm{mmol} / \mathrm{l}) \\
+\mathrm{MI}(2 \mathrm{mmol} / \mathrm{l}) \\
+\mathrm{MI}(4 \mathrm{mmol} / \mathrm{l}) \\
+\mathrm{MI}(9 \mathrm{mmol} / \mathrm{l})\end{array}$ & $\begin{array}{l}12 \\
49 \\
16\end{array}$ & $\begin{array}{r}4^{\mathrm{b}} \\
27^{\mathrm{c}} \\
5^{\mathrm{b}}\end{array}$ & $\begin{array}{l}33 \\
55 \\
31\end{array}$ \\
\hline $\begin{array}{l}\text { D-glucose }(44 \mathrm{mmol} / \mathrm{l}) \\
+\mathrm{MI}(4 \mathrm{mmol} / \mathrm{l}) \\
+ \text { indomethacin }(40 \mu \mathrm{mol} / \mathrm{l})\end{array}$ & 18 & $5^{b, d}$ & 28 \\
\hline
\end{tabular}

Both B10.A and CD-1 strains were maintained on a $12-\mathrm{h}$ light cycle with the dark phase extending from 05.00 to 17.00 hours. Females were mated with syngeneic males from 10.00 to 16.00 hours. Presomite embryos $\left(8^{1 / 4}\right.$ day B10.A, 8 day CD-1; plug day = day 0$)$ were explanted and cultured using techniques described earlier [17]. The culture medium consisted of immediately-centrifuged, heatinactivated rat serum and $1 \%$ penicillin-streptomycin mixture (5000 I.U./Mg each per ml; Flow Laboratories McLean, VA, USA). Additions to the culture medium were made at the expense of appropriate volumes of rat serum. Glucose (D- or L-) in distilled water was included as a $3 \%$ addition and $\mathrm{MI}$ in distilled water was included as a $2 \%$ addition. Indomethacin and the prostaglandins were solubilized in absolute ethanol and included as $0.33 \%$ and $0.1 \%$ additions, respectively. Controls received appropriate volumes of water and ethanol. The embryos were collected from culture at $48 \mathrm{~h}$. The extraembryonic membranes (visceral yolk sac, amnion and ectoplacental cone) were removed and the rostral neural tube was determined to be either open or fused. Sadler et al. [18] identified several stages of rostral neural tube closure prior to fusion. To simplify presentation, all of these pre-fusion end points are grouped together as neural tube defects [10] and included in the open category. The embryonic heartbeat was used as a measure of viability.

Glucose (D- and L-), MI, indomethacin, and prostaglandins $\mathrm{E}_{2}$ $\left(\mathrm{PGE}_{2}\right)$ and $\mathrm{F}_{2 \alpha}\left(\mathrm{PGF}_{2 \alpha}\right)$ were obtained from Sigma Chemical Company St. Louis, MO, USA. Carba-prostacyclin $\left(\mathrm{CPGI}_{2}\right)$, the stable analogue of prostacyclin $\left(\mathrm{PGI}_{2}\right)$, was purchased from Cayman Chemical Company Ann Arbor, MI, USA.

\section{Statistical analysis}

Statistical comparisons between groups were performed by the Pearson chi-square method; $p$-values $>0.05$ were considered not significant (NS).

\section{Results}

Table 1 summarizes the results of the MI supplementation and the MI plus indomethacin experiments. Rostral neural tube fusion occurred significantly $(p<0.001)$ less frequently in embryos treated with D-glucose than in controls. When MI at a concentration of $4 \mathrm{mmol} / \mathrm{l}$ was added to the $\mathrm{D}$-glucose $(44 \mathrm{mmol} / \mathrm{l})$ medium, a significant $(p=0.02)$ protective effect was seen when compared to $D$ glucose alone. However, this protective effect was also significantly $(p<0.01)$ different than the control and therefore incomplete. Lower $(2 \mathrm{mmol} / \mathrm{l})$ and higher $(9 \mathrm{mmol} / \mathrm{l})$ concentrations of MI were ineffective in protecting against the inhibition of rostral neural tube closure caused by elevated D-glucose levels.

The protective effect of MI was reversed in experiments when indomethacin $(140 \mu \mathrm{mol} / \mathrm{l})$ was added to medium containing both $\mathrm{D}$-glucose and the protective concentration of MI. Indomethacin permitted rostral neural tube fusion in only $28 \%$ of the embryos, a percentage statistically the same as D-glucose alone, but significantly different $(p<0.05)$ than D-glucose plus MI.

Since indomethacin at the concentration used is inhibitory at various sites in arachidonic acid mobilization and metabolism including the cyclooxygenase step [19], the above data suggested that prostaglandins might be important in the process of neural tube fusion. Experiments were therefore carried out to test the effect of prostaglandins in reversing glucose-induced failure of neural tube fusion. The results are presented in Figure 1. In these studies, fusion percentages for L-glucose controls were clearly higher than D-glucose-treated embryos $(p<0.001)$. The addition to the $\mathrm{D}$-glucose medium of $\mathrm{PGE}_{2}$ at a concentration of $142 \mathrm{nmol} / \mathrm{l}$ completely protected the embryos against the failure of neural tube fusion seen in the presence of excess glucose ( $p=$ NS vs L-glucose controls and $p<0.02$ vs D-glucose). $\mathrm{PGE}_{2}$ at $284 \mathrm{nmol} / 1$ and $\mathrm{cPGI}_{2}$ at $143 \mathrm{nmol} / \mathrm{l}$ and $285 \mathrm{nmol} / \mathrm{l} \mathrm{par}-$ tially, though not significantly, protected, and each of these prostaglandins at the highest concentration (2837 $\mathrm{nmol} / 1 \mathrm{PGE}_{2} ; 2835 \mathrm{nmol} / \mathrm{cPGI}$ was slightly more teratogenic than D-glucose alone. Low concentrations of $\mathrm{PGE}_{2}(28 \mathrm{nmol} / \mathrm{l})$ and $\mathrm{cPGI}_{2}(29 \mathrm{nmol} / \mathrm{l})$ did not afford protection against failure of neural tube fusion, $\mathrm{PGF}_{2 \alpha}$ was least potent. While $282 \mathrm{nmol} / \mathrm{l}$ of $\mathrm{PGF}_{2 \alpha}$ was more effective than $141 \mathrm{nmol} / \mathrm{l}$, neither concentration significantly reversed the failure of rostral neural tube fusion associ-

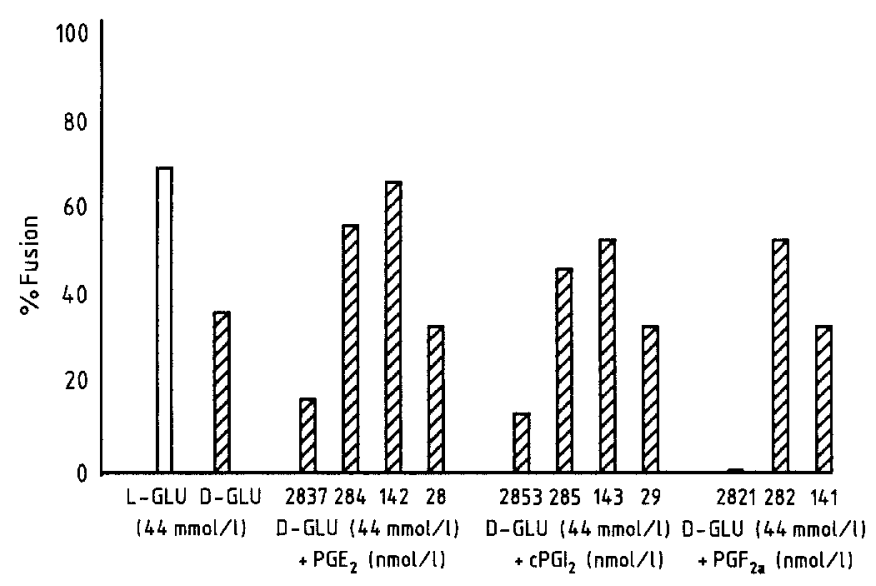

Fig.1. Reversal by prostaglandins of $D$-glucose inhibited neural tube fusion. Numbers of embryos per category are as follows: L-glucose, 70; D-glucose, 54; $\mathrm{PGE}_{2}, 12-19 ; \mathrm{cPGI}_{2}, 10-23$ and; $\mathrm{PGF}_{2 \alpha}, 10-$ 15. As a measure of growth, crown-rump lengths after $48 \mathrm{~h}$ of culture were identical for L-glucose controls $(2.4 \pm 0.3 \mathrm{~mm})$ and D-glucosetreated embryos $(2.4 \pm 0.6 \mathrm{~mm})$. Similarly, crown-rump measurements for most of the prostaglandin-treated embryos were statistically the same as controls. Exceptions include significantly smaller embryos treated with teratogenic concentrations of $\mathrm{CPGI}_{2}$ $(2853 \mathrm{nmol} / \mathrm{l})$ or $\mathrm{PGF}_{2 \alpha}(2821 \mathrm{nmol} / \mathrm{l})$ and, inexplicably, smaller embryos in the $\mathrm{PGE}_{2}(28 \mathrm{nmol} / \mathrm{l})$ category 
ated with high levels of $\mathrm{D}$-glucose; moreover, $\mathrm{PGF}_{2 \alpha}$ at $2821 \mathrm{nmol} / 1$ completely prevented fusion $(p<0.02$ vs D-glucose and $p=0.0001$ vs L-glucose).

\section{Discussion}

The supplementation of MI in suitable animal models has previously been shown to modify abnormalities caused by diabetes in peripheral nerve (decreased motor nerve conduction velocity [20]), retina (decreased amplitude of the electroretinogram C-wave [21]) and kidney (increased glomerular filtration rate [22]). In the present study, the data demonstrate that supplemental MI exerts a significant protective effect against the teratogenic effects of a high glucose concentration on neural tube fusion. Specifically, the addition of MI to the mouse embryo cultured in the presence of excess D-glucose was associated with a significant, albeit incomplete, protection against failure of neural tube fusion. This protection against glucose-induced neural tube defects afforded by MI supplementation is consistent with data presented by Sussman and Matschinsky [23], demonstrating that normal developmental MI increases are prevented in the neuroepithelium of embryos obtained from streptozotocin-induced diabetic rats. The concentration dependent effects of MI seen in our study are in accord with observations by Greene et al. [24] showing that MI supplementation ameliorates impaired motor nerve conduction velocity in diabetic rats only within a critical concentration range.

The protection against glucose-related failure of neural tube fusion provided by supplementation of MI can be reversed when indomethacin, an inhibitor of arachidonic acid metabolism, is added to the experimental system. These studies were performed because we had previously published data to show that arachidonic acid supplementation significantly protected against glucose-induced failure of normal rostral neural tube fusion [17]. The suggestion that arachidonic acid was involved in neural tube fusion was confirmed by Pinter et al. [25]. In a subsequent paper [26], this group once again confirmed that arachidonic acid protected against glucose-induced neural tube defects, but they were not able to document differences in the concentration of arachidonic acid between control and hyperglycaemia-treated whole embryos. This is perhaps not surprising, since a functional deficiency of arachidonic acid in one specific part of the embryo during neurulation (the head) may not be reflected in an overall change in its concentration in the whole embryo. Additionally, they measured arachidonic acid concentrations only at the end of the culture period and thereby may have missed specific but short-lived changes occurring during the critical time of neural tube fusion.

The reversal of the MI protective effect by indomethacin suggested that prostaglandins may play a specific role in neural tube fusion. This was tested directly by the addition to the culture medium of $\mathrm{PGE}_{2}, \mathrm{PGF}_{2 \alpha}$, and $\mathrm{cPGI}_{2}$. The choice of these prostaglandins was based on data demonstrating that $\mathrm{PGE}_{2}, \mathrm{PGF}_{2 \alpha}$ and 6-keto- $\mathrm{PGF}_{1 \alpha}$, the stable metabolite of prostacyclin $\left(\mathrm{PGI}_{2}\right)$, are synthesized in cells from embryonic rats and mice $[27,28]$ and the em- bryonic ewe [29]. As shown in Figure 1, $\mathrm{PGE}_{2}$ is most potent in affording protection; $\mathrm{CPGI}_{2}$ and $\mathrm{PGF}_{2 \alpha}$ only partially protect. The prostaglandin concentrations which are effective in these studies are within the normal working concentration range of prostaglandins in tissue culture media $[30,31]$. The finding that high concentrations ( $>2800 \mathrm{nmol} / \mathrm{l}$ ) of the prostaglandins are teratogenic is consistent with the observation that prostaglandins injected during pregnancy can cause congenital malformations in mice [32].

In summary, data are presented in this paper to document that MI supplementation in the mouse embryo culture system affords significant protection against glucoseinduced failure of rostral neural tube fusion. The protective effects of MI are reversed when indomethacin is placed in the culture medium in addition to D-glucose and MI. The indomethacin experiments suggest that prostaglandins may be involved in neural tube fusion. $\mathrm{PGE}_{2}$, used in a concentration which has been shown in other systems to elicit biological responses, completely protects against the glucose-related malformation. Taken together, the data are compatible with the hypothesis that the failure of rostral neural tube fusion seen in the mouse embryo culture system of diabetic embryopathy is mediated through a mechanism involving abnormalities in both the MI and arachidonic acid pathways, a result of which is a deficiency of prostaglandins at a critical time of neural tube fusion.

Acknowledgements. We wish to thank Ms. J.Dolhancryk and Ms. E. Alicea-Cruz for preparing the manuscript. This work was supported in part by the Philadelphia affiliate of the American Diabetes Association, and the Virgil Kauffman Foundation, and by grants from the National Institutes of Health (P30-DK19525) and the Juvenile Diabetes Foundation (188904).

\section{References}

1. Mills JL (1982) Malformations in infants of diabetic mothers. Teratology 25: 385-394

2. Small L, Cassidy L, Leiper JM, Paterson KR, Lunan CB, MacCuish AC (1986) Outcome of pregnancy in insulin-dependent (Type I) diabetic women between 1971 and 1984. Q J Med 61: 1159-1169

3. Soler NG, Walsh CH, Malins JM (1976) Congenital malformations in infants of diabetic mothers. Q J Med 45:303-313

4. Freinkel N (1980) The Banting lecture 1980: of pregnancy and progeny. Diabetes 29: 1023-1035

5. Eriksson UJ, Dahlstrom VE, Styrud J (1985) Metabolicallydetermined teratogenesis: malformations and maternal diabetes. Trans Biochem Soc 13: 79-82

6. Slourish M, Morris GM (1977) Glycosaminoglycan synthesis in rat embryos during the formation of the primary mesenchyme and neural folds. Dev Biol 57:75-86

7. Horton WE, Sadler TW (1983) Effects of maternal diabetes on early embryogenesis: alterations in morphogenesis produced by the ketone body, B-hydroxybutyrate. Diabetes 32:610-616

8. Balkin W, Phillips LS, Goldstein S, Sadler TW (1988) A potential role of somatomedin inhibitors in the production of diabetic embryopathies. Teratology 37:271-282

9. Freinkel N, Cockroft DL, Lewis N, Gorman L, Akazawa S, Phillips LS, Shambaugh GE III (1986) The 1986 McCollum Award Lecture. Fuel-mediated teratogenesis during early organogenesis: the effects of increased concentrations of glucose, ketones 
or somatomedin inhibitor during rat embryo culture. Am J Clin Nutr 44: 986-995

10. Sadler TW, Hunter ES III, Wynn RE, Phillips LS (1989) Evidence for multifactorial origin of diabetes-induced embryopathies. Diabetes 38: 70-74

11. Eriksson UJ, Naeser P, Brolin SE (1986) Increased accumulation of sorbitol in offspring of manifest diabetic rats. Diabetes 35 : $1356-1363$

12. Hod M, Star S, Passonneau JV, Unterman TG, Freinkel N (1986) Effect of hyperglycemia on sorbitol and myo-inositol content of cultured rat conceptus: failure of aldose reductase inhibitors to modify myo-inositol depletion and dysmorphogenesis. Biochem Biophys Res Comm 140: 974-980

13. Winegrad AI (1987) Does a common mechanism induce the diverse complications of diabetes? Diabetes 36: 396-406

14. Greene DA (1986) A sodium pump defect in diabetic peripheral nerve corrected by sorbinil administration: relationship to myoinositol metabolism and nerve conduction slowing. Metabolism 35 [Suppl 1]: 60-65

15. MacGregor LC, Matschinsky FM (1986) Experimental diabetes mellitus impairs the function of the retinal pigmented epithelium. Metabolism 35 [Suppl 1]: 28-34

16. Cohen M (1986) Aldose reductase, glomerular metabolism and diabetic nephropathy. Metabolism 35 [Suppl 1]: 55-59

17. Goldman AS, Baker L, Piddington RL, Marx B, Herold R, Egler J (1985) Hyperglycemia-induced teratogenesis is mediated by a functional deficiency of arachidonic acid. Proc Natl Acad Sci USA 82: 8227-8231

18. Sadler TW, Horton WE, Warner CW (1982) Whole embryo culture: a screening technique for teratogens? Teratogenesis Carcinog Mutagen 2: 243-253

19. Kaplan L, Weiss J, Elsbach P (1978) Low concentrations of indomethacin inhibit phospholipase $\mathrm{A}_{2}$ of rabbit polymorphonuclear leukocytes. Proc Natl Acad Sci USA 75: 2955-2958

20. Greene DA, Lattimer S, Ulbrect BS, Carrol P (1985) Glucoseinduced alterations in nerve metabolism: current perspective on the pathogenesis of diabetic neuropathy and future directions for research and therapy. Diab Care 8:290-299

21. MacGregor LC, Matschinsky FM (1985) Treatment with aldose reductase inhibitor or with myo-inositol arrests deterioration of the electroretinogram of diabetic rats. J Clin Invest 76: 887-889

22. Goldfarb S, Simmons DA, Kern EFO (1986) Amelioration of glomerular hyperfiltration in experimental diabetes by myo-inositol supplementation and sorbinil. Trans Am Assoc Phys 99: 6772
23. Sussman I, Matschinsky FM (1988) Diabetes affects sorbitol and myo-inositol levels of neuroectodermal tissue during embryogenesis in rat. Diabetes 37: 974-981

24. Greene DA, DeJesus PV Jr, Winegrad AI (1975) Effects of insulin and dietary myoinositol on impaired peripheral motor nerve conduction velocity in acute streptozotocin diabetes. J Clin Invest 55: 1326-1336

25. Pinter E, Reece EA, Czaba ZL, Garcia-Segura M, Hobbins JC, Mahoney MJ, Naftolin F (1986) Arachidonic acid prevents hyperglycemia-associated yolk sac damage and embryopathy. Am J Obstet Gynecol 155: 691-702

26. Pinter E, Reece EA, Ogburn PL, Turner S, Hobbins JC, Mahoney MJ, Naftolin F (1988) Fatty acid content of yolk sac and embryo in hyperglycemia-induced embryopathy and effect of arachidonic acid supplementation. Am J Obstet Gynecol 159: $1484-1490$

27. Klein KL, Scott WJ Jr, Clark KE (1981) Measurement of prostaglandins in embryonic tissue using radioimmunoassay. Prostaglandins 22: 623-630

28. Anton RF, Randall CL, Becker HC (1988) PGE measurement in mouse embryos and uterine/embryo tissue. Prostaglandins 36 : 835-846

29. Rawlings NC, Hyland JH (1985) Prostaglandin F and E levels in the conceptus, uterus and plasma during early pregnancy in the ewe. Prostaglandins 29:933-951

30. Kunkel SL, Spengler M, May MA, Spengler R, Larrick J, Remick D (1988) Prostaglandin $E_{2}$ regulates macrophage-derived tumor necrosis factor gene expression. J Biol Chem 263: 53805384

31. Negrel R, Gaillard D, Ailhaud G (1989) Prostacyclin as a potent effector of adipose-cell differentiation. Biochem J 257:399 - 405

32. Persaud TVN (1975) The effects of prostaglandin $E_{2}$ on pregnancy and embryonic development in mice. Toxicology 5: 97-101

Received: 5 December 1989

and in revised form: 16 May 1990

Dr. L.Baker

Division of Endocrinology/Diabetes

The Children's Hospital of Philadelphia

34th \& Civic Center Boulevard

Philadelphia, PA 19104

USA 